Open Access

\title{
Association of HSD11B1 polymorphic variants and adipose tissue gene expression with metabolic syndrome, obesity and type 2 diabetes mellitus: a systematic review
}

Filipe Valvassori do Nascimento ${ }^{2}$, Vanessa Piccoli², Mayara Abichequer Beer ${ }^{2}$, Anize Delfino von Frankenberg ${ }^{2}$, Daisy Crispim ${ }^{1,2}$ and Fernando Gerchman ${ }^{1,2^{*}}$

\begin{abstract}
The HSD11B1 gene is highly expressed in abdominal adipose tissue, and the enzyme it encodes catalyzes the interconversion of inactive cortisone to hormonally active cortisol. Genetic abnormalities of HSD11B1 have been associated with the development of abnormal glucose metabolism and body fat distribution. To systematically review studies evaluating the association of HSD11B1 gene expression in abdominal adipose tissue and HSD11B1 polymorphisms with obesity, the metabolic syndrome (MetS), and type 2 diabetes (T2DM), we conducted a search in MEDLINE, SCOPUS, and Cochrane Library databases in April 2015. The inclusion criteria were observational studies (cross-sectional, cohort, or case-control), conducted in adults, which analyzed the relationship of HSD11B1 polymorphisms and/or HSD11B1 expression in abdominal adipose tissue with obesity, MetS, or T2DM. Of 802 studies retrieved, 32 met the inclusion criteria (23 gene expression and 9 polymorphism studies). Twenty one studies analyzed the relationship between abdominal subcutaneous and/or visceral HSD11B1 expression with central and/or generalized obesity. Most studies reported that abdominal adipose HSD11B1 expression increased with increasing body mass index (15 studies) and abnormalities of glucose metabolism (7 studies), and varied with the presence of MetS (3 studies). Nine studies analyzed the association of 26 different HSD11B1 polymorphic variants with obesity, MetS, and T2DM. Only an Indian study found an association between a polymorphic variant at the HSD11B1 gene with MetS whereas in Pima Indians another polymorphic variant was found to be associated with T2DM. While the literature suggests that HSD11B1 is hyperexpressed in abdominal adipose tissue in subjects with obesity and abnormal glucose metabolism, this seems to be not true for HSD11B1 gene expression and MetS. Although an association of polymorphic variants of HSD11B1 with MetS in Indians and in the T2DM population of Pima Indians were found, most studies did not find a relationship between genetic polymorphic variants of HSD11B1 and obesity, MetS, and T2DM. Their reported conflicting and inconclusive results, suggesting that polymorphic variants of HSD11B1 may have only a small role in the development of metabolic abnormalities of susceptible populations in the development of MetS and T2DM.
\end{abstract}

Keywords: Diabetes mellitus, Obesity, Metabolic syndrome, HSD11B1, Polymorphisms, Gene expression

\footnotetext{
* Correspondence: fgerchman@gmail.com

'Division of Endocrinology, Hospital de Clínicas de Porto Alegre, Rua Ramiro

Barcelos 2350, Prédio 12, $4^{\circ}$ andar, Bairro Santana, Porto Alegre, RS

90035-003, Brazil

${ }^{2}$ Postgraduate Program in Medical Sciences: Endocrinology, Faculdade de Medicina, Universidade Federal do Rio Grande do Sul, Rua Ramiro Barcelos 2400, $2^{\circ}$ andar, PPG Endocrinologia, Bairro Santana, Porto Alegre, RS 90035-003, Brazil
}

\section{Biomed Central}

(c) 2015 do Nascimento et al. This is an Open Access article distributed under the terms of the Creative Commons Attribution License (http://creativecommons.org/licenses/by/4.0), which permits unrestricted use, distribution, and reproduction in any medium, provided the original work is properly credited. The Creative Commons Public Domain Dedication waiver (http:// creativecommons.org/publicdomain/zero/1.0/) applies to the data made available in this article, unless otherwise stated. 


\section{Introduction}

Hydroxysteroid (11-beta) dehydrogenase type 1 (11$\beta H S D 1)$ is a bidirectional enzyme, encoded by the HSD11B1 gene, that is highly expressed in liver and adipose tissue. It normally converts the inactive hormone cortisone into its active form cortisol, acting as a reductase [1].

Overexpression of the HSD11B1 gene in adipocytes has been shown to be related to high cortisol concentrations in adipose tissue and to the development of central obesity, insulin resistance (IR), and diabetes in mouse models [2]. On the other hand, HSD11B1 knockout mice exposed to a high fat diet are protected against the development of obesity and hyperglycemia [3]. Moreover, $11-\beta H S D 1$ inhibitors have been shown to be effective in treating different aspects of the metabolic syndrome (MetS), promoting weight loss and reducing IR and hyperglycemia.

Cushing's syndrome, which is caused by excess glucocorticoid production, has clinical features that resemble those of MetS in several aspects, suggesting that they share possible pathogenic pathways which result in their metabolic abnormalities. As overexpression of HSD11B1 in abdominal adipose tissue is associated with increased adipose tissue cortisol concentrations, polymorphic variants of this gene may be related to MetS development [4].

To elucidate this issue, we conducted a systematic review of the literature addressing the potential association of $H S D 11 B 1$ polymorphic variants and abdominal HSD11B1 adipose tissue expression with MetS, type 2 diabetes mellitus (T2DM), and obesity.

\section{Methods}

The Preferred Reporting Items for Systematic Reviews and Meta-Analysis (PRISMA) statement was used in this report. This systematic review is registered in PROSPERO with number CRD42014008705 and can be assessed on http://www.crd.york.ac.uk/PROSPERO/display_record.asp? ID=CRD42014008705\#.VQmwC9LF9A0.

\section{Search strategy, study selection, and data extraction}

Observational studies (cross-sectional, cohort, or case-control) which have analyzed the relationship of polymorphisms and/or HSD11B1 gene expression with obesity, MetS, or T2DM in human adults were considered eligible. Exclusion criteria were: subjects under 18 years, the presence of malignancy, infectious diseases or diseases that may affect $H S D 11 B 1$ gene expression or was described to be associated with its polymorphic variants such as Cushing's syndrome, osteoporosis, cortisone reductase deficiency (CRD), Alzheimer's disease and polycystic ovary syndrome (PCOS). A literature search was performed in MEDLINE, Cochrane Central, and Scopus databases. We also manually searched the references of published studies. Study selection was not limited by language. The search strategy was based on the following Medical Subject Headings (MeSH): (((“"Obesity"[Mesh]) OR "Diabetes Mellitus, Type 2"[Mesh]) OR "Prediabetic State"[Mesh])) AND ("11-beta-Hydroxysteroid Dehydrogenase Type 1"[Mesh]).

Study selection was performed by two independent investigators (FVN and VP). Disagreements were resolved by discussion and consensus; when necessary, a third reviewer (FG) was consulted. Data was extracted by two investigators (FVN and VP) using a standardized form. The information extracted from each individual study was as follows: design, ethnicity, polymorphisms genotyped, tissue site for biopsy and gene expression analysis, number of individuals in each group, gender distribution, body mass index (BMI), waist circumference, waistto-hip ratio, and fasting and 2-h plasma glucose levels after a 75-g oral glucose tolerance test. The NewcastleOttawa Scale (NOS) was used to assess the quality of the selected studies [5]. The NOS contains eight items categorized into three dimensions, including selection, comparability, and exposure. A series of response options is provided for each item. A star scoring system is used to allow semi quantitative assessment of study quality, such that the highest-quality studies are awarded a maximum of one star for each item, except on the comparability item, for which two stars can be assigned. The total NOS score thus ranges from zero to nine stars.

\section{Results}

Our search strategy yielded 802 records (Figure 1). On the basis of titles and abstracts, we selected 71 studies for full-text examination, of which 32 fulfilled the final inclusion criteria. Studies were grouped according to the relationship they set out to assess: 1) HSD11B1 gene expression in subcutaneous/visceral abdominal fat and MetS [6-8] ; 2) HSD11B1 gene expression in subcutaneous/visceral abdominal fat and hyperglycemia [6,8-14]; 3) $H S D 11 B 1$ gene expression in subcutaneous/visceral abdominal fat and obesity [4,6-11,13,15-28]; 4) HSD11B1 polymorphisms and obesity, MetS, or T2DM [29-37]. The main results of these studies are presented in Tables 1, 2, 3 and 4. The mean quality score was 5.31 stars, and the details of quality assessment were described in Table 5.

\section{Relationship between HSD11B1 expression in subcutaneous and visceral abdominal fat and the metabolic syndrome}

Three studies compared the expression of HSD11B1 in adipose tissue according to the presence of MetS [6-8] (Table 1). These studies included a total of 121 participants (mean age, 41.7 years). Two of them recruited only obese subjects, whereas the third compared obese vs. 


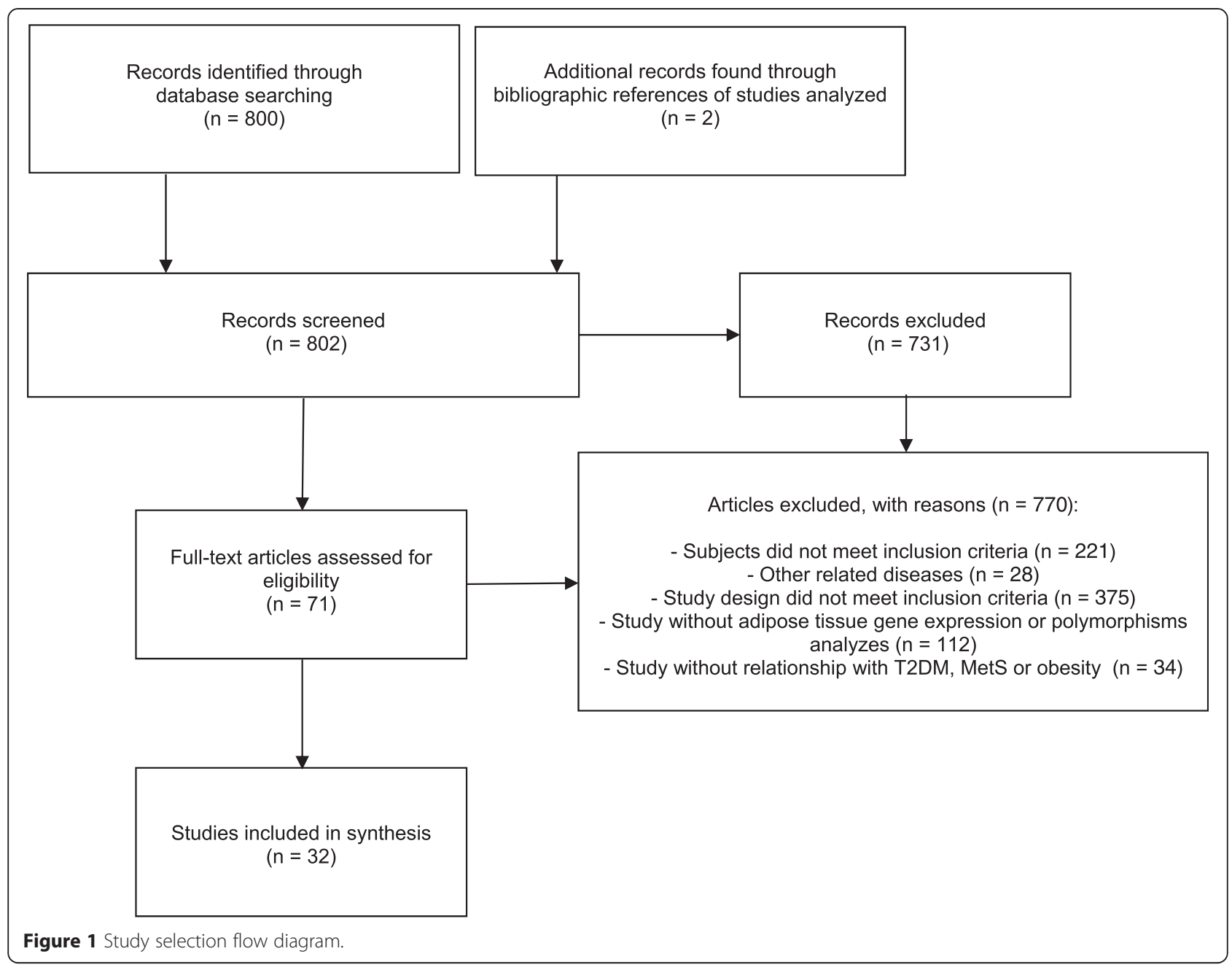

lean control subjects; two studies recruited subjects with a predominant female population, whereas one included only women. HSD11B1 expression was quantified as arbitrary units using similar methodology (RT-qPCR), differing primarily in their housekeeping genes (GUSB, TBP and 18S). Prevalence of T2DM was low and varied between 7.4 and $19.4 \%$. In the first study [6], HSD11B1 expression in abdominal subcutaneous adipose tissue (SAT) was higher in 62 obese subjects with MetS compared to those without MetS, whereas expression in abdominal visceral adipose tissue (VAT) was similar between groups. Contrasting with these findings, another study with 32 obese individuals (six with T2DM) [8] found that HSD11B1 expression was not significantly higher in either abdominal SAT or VAT in subjects without MetS compared to those with MetS. On the other hand, in a case-control study, HSD11B1 expression in abdominal VAT was significantly higher in 19 obese women (two with T2DM) than in eight lean women [7] (Table 1).
Relationship between HSD11B1 expression in subcutaneous and visceral abdominal fat and hyperglycemia

Eight studies analyzed the relationship between HSD11B1 expression in abdominal SAT and VAT with estimates of insulin resistance, $\beta$-cell function, blood glucose levels and abnormalities of glucose metabolism and T2DM, in populations of different ethnic background, including Caucasians, African Americans, Pima Indians, Hispanic Americans and populations from Sweden and Denmark (data not clear about ethnicity) (Table 2). One study included only women [11] and seven studies included both genders, mostly women in 6 and mostly men in one $[6,8-10,12-14]$. The prevalence of T2DM varied between 0 and $22.4 \%$ among studies, with two studies where this information was not provided. These differences in gender distribution among studies do not affect the results of these findings. While HSD11B1 VAT expression increased respectively with increasing fasting plasma glucose [9] and HOMA-IR [10] in morbid obese subjects of two different studies, two have 
Table 1 Relationship between HSD11B1 gene expression in abdominal adipose tissue and the metabolic syndrome: studies and subjects' characteristics

\begin{tabular}{|c|c|c|c|c|c|c|c|}
\hline Author, year & Subjects' characteristics & T2DM & Obesity & BMI $\left(\mathrm{kg} / \mathrm{m}^{2}\right)$ & MetS & Expression in VAT (AU) & Expression in SAT (AU) \\
\hline \multirow[t]{4}{*}{ Alberti, 2007 [6] } & $\mathrm{n}: 62$ & & & & & & \\
\hline & Female: 52 (83.9) & $12(19.4)$ & $62(100.0)$ & $37.4 \pm 5.1$ & $25(40.3)$ & MetS+: $2.6 \pm 0.5$ & MetS+: $2.0 \pm 1.5$ \\
\hline & Age: $44.4 \pm 11.1$ & & & & & MetS-: $1.7 \pm 1.0$ & MetS-: $0.7 \pm 0.4^{\mathrm{a}}$ \\
\hline & Ethnicity: N/D & & & & & & \\
\hline \multirow[t]{4}{*}{ Muñoz, 2009 [8] } & n: 32 & & & & & & \\
\hline & Female: 21 (65.6) & $6(18.8)$ & $32(100.0)$ & $36.7 \pm 3.8$ & $16(50.0)$ & MetS+: 7.5 & MetS+: 11.6 \\
\hline & Age: $40.2 \pm 12.3$ & & & & & MetS-: 10.3 & MetS-: 12.3 \\
\hline & Ethnicity: N/D & & & & & & \\
\hline \multirow[t]{4}{*}{ Michalaki, 2012 [7] } & $n=27$ & & & & & & \\
\hline & Female: 27 (100) & $2(7.4)$ & $19(70.4)$ & $46.1 \pm 6.6$ & $11(40.7)$ & Lean controls: $27.8 \pm 16.0$ & MetS+: $86.5 \pm 29.8$ \\
\hline & Age: $37.3 \pm 9.7$ & & & & & MetS+: $62.9 \pm 24.4$ & MetS-: $155.9 \pm 124.9$ \\
\hline & Ethnicity: N/D & & & & & MetS-: $107.2 \pm 77.7^{b}$ & \\
\hline
\end{tabular}

Data expressed as absolute and relative frequencies or mean \pm standard deviation as appropriate. T2DM: type 2 diabetes mellitus; BMI: body mass index; MetS: metabolic syndrome; VAT: visceral adipose tissue; SAT: subcutaneous adipose tissue; AU: arbitrary units; N/D: not described. ${ }^{\text {a }}$ < < 0.01 vs. MetS+ group. ${ }^{\text {bP }}<0.01$ vs. lean controls.

shown similar results with abdominal SAT $[11,13]$. While comparing the expression of abdominal SAT HSD11B1 according to glucose tolerance, additional studies have shown that this expression was greater respectively in those with T2DM $(1.8 \pm 0.7$ vs. $1.1 \pm$ 0.4 AU; $\mathrm{P}<0.05)$ [6] and those with impaired glucose tolerance $(0.5 \pm 0.06$ vs. $0.29 \pm 0.03 \mathrm{AU} ; \mathrm{P}<0.005)$ compared with normal controls [14]. Although in another study, increased abdominal SAT HSD11B1 expression was related with decreased beta-cell function $(r=-0.574)$ estimated by the disposition index while adjusting for different confounders, this relationship became not significant after the inclusion of hepatic fat fraction in the multivariate model [12]. Finally, one study showed a trend toward increased expression of HSD11B1 in abdominal SAT in subjects with T2DM compared to normal controls, although the statistical significance of this comparison was not shown due to the small number of subjects with T2DM $(n=6)$ [8].

\section{Relationship between HSD11B1 expression in} subcutaneous and visceral abdominal fat and obesity

Twenty one studies assessed the potential relationship of HSD11B1 expression in subcutaneous and visceral abdominal fat with obesity (Table 3 ). Six studies did not find a relationship between $H S D 11 B 1$ expression in abdominal SAT or VAT and central or generalized obesity $[6,7,9,10,24,26]$. Conversely, in two studies [15,17], abdominal SAT and VAT HSD11B1 expression was higher in obese women than in lean women. Similar results were reported in other three studies assessing HSD11B1 expression only in abdominal SAT of female subjects $[16,18,25]$. In the first of these studies [15], a greater gene expression in abdominal SAT was also associated with a greater waist circumference and percentage of body fat. In two other studies, HSD11B1 gene expression was higher in the abdominal VAT of obese subjects than in lean subjects $[19,20]$. In four studies $[8,11,13,27] H S D 11 B 1$ expression in abdominal SAT was positively related with measures of body size and central obesity, namely respectively, BMI $[8,13,27]$ and intra-abdominal VAT volume estimated by MRI [11]. In other two studies [21,22], HSD11B1 expression in abdominal SAT was higher in obese men and women than in non-obese subjects. In another study, the expression of HSD11B1 in abdominal VAT was higher in obese women than in lean controls, but this relationship was not assessed in men due to small sample size. Zha et al. found abdominal VAT and SAT HSD11B1 expression higher in obese subjects than in lean controls as well as a positive correlation between these expressions with BMI [28]. Finally, in a small study sample of women $(\mathrm{n}=17)$, it was found a positive correlation between abdominal SAT HSD11B1 expression and waist circumference $(\mathrm{r}=0.53 ; \mathrm{P}<0.05)$, but not with $\mathrm{BMI}$ [23]. 
Table 2 Relationship between HSD11B1 gene expression in abdominal adipose tissue and hyperglycemia: studies and subjects' characteristics

\begin{tabular}{|c|c|c|c|c|c|c|c|}
\hline Author, year & $\begin{array}{l}\text { Subjects' } \\
\text { characteristics }\end{array}$ & T2DM & Obesity & BMI $\left(\mathrm{kg} / \mathrm{m}^{2}\right)$ & MetS & Expression in VAT & Expression in SAT \\
\hline \multirow[t]{5}{*}{ Lindsay, 2003 [13] } & n: 31 & & & & & & \\
\hline & Female: 14 (45.2) & \multirow[t]{4}{*}{ N/D } & \multirow[t]{4}{*}{$\mathrm{N} / \mathrm{D}$} & \multirow[t]{4}{*}{$35.4 \pm 7.4$} & \multirow[t]{4}{*}{$\mathrm{N} / \mathrm{D}$} & \multirow[t]{4}{*}{ N/D } & \multirow{4}{*}{$\begin{array}{c}r=0.46, P<0.05 \text { with } \\
\text { HOMA-IR }\end{array}$} \\
\hline & Age: $28.8 \pm 7.7$ & & & & & & \\
\hline & Ethnicity: 12 Caucasian & & & & & & \\
\hline & 19 Pima Indian & & & & & & \\
\hline \multirow[t]{6}{*}{ Goedecke, 2006 [11] } & $\mathrm{n}: 26$ & & & & & & \\
\hline & Female: 26 (100) & \multirow[t]{5}{*}{$0(0)$} & \multirow[t]{5}{*}{ N/D } & \multirow[t]{5}{*}{$27.2 \pm 0.9$} & \multirow[t]{5}{*}{$N / D$} & \multirow{5}{*}{$\begin{array}{l}r=-0.13, P=N S \\
\text { with fasting glucose }\end{array}$} & \multirow{5}{*}{$\begin{aligned} r= & 0.40, P<0.016 \text { with } \\
& \text { fasting glucose }\end{aligned}$} \\
\hline & Age: $41 \pm 2.0$ & & & & & & \\
\hline & Ethnicity: 11 Caucasian & & & & & & \\
\hline & 8 Mixed race & & & & & & \\
\hline & 7 Black & & & & & & \\
\hline \multirow[t]{4}{*}{ Alberti, 2007 [8] } & n: 62 & & & & & & \\
\hline & Female: 52 (83.9) & \multirow[t]{3}{*}{$12(19.4)$} & \multirow[t]{3}{*}{$62(100.0)$} & \multirow[t]{3}{*}{$37.4 \pm 5.1$} & \multirow[t]{3}{*}{$25(40.3)$} & \multirow[t]{3}{*}{$\mathrm{N} / \mathrm{D}$} & \multirow{3}{*}{$\begin{array}{l}r=0.460, P<0.0001 \text { with } \\
\quad \text { fasting glucose }\end{array}$} \\
\hline & Age: $44.4 \pm 11.1$ & & & & & & \\
\hline & Ethnicity: N/D & & & & & & \\
\hline \multirow[t]{4}{*}{ Tomlinson, 2008 [14] } & $\mathrm{n}=101$ & & & & & & \\
\hline & Female: 66 (65.3) & \multirow[t]{3}{*}{$7(6.9)$} & \multirow[t]{3}{*}{$101(100)$} & \multirow[t]{3}{*}{$34.4 \pm 4.3$} & \multirow[t]{3}{*}{$N / D$} & \multirow[t]{3}{*}{ N/D } & $r=0.44, P<0.005$ with \\
\hline & Age: $48.0 \pm 7.0$ & & & & & & \\
\hline & Ethnicity: N/D & & & & & & \\
\hline Muñoz, 2009 [8] & n: 32 & & & & & & \\
\hline & Female: 21 (65.6) & $6(18.8)$ & $32(100.0)$ & $36.7 \pm 3.8$ & $16(50.0)$ & $r=-0.19, P=N S$ & $r=0.15, P=N S$ with \\
\hline & Age: $40.2 \pm 12.3$ & & & & & & \\
\hline & Ethnicity: N/D & & & & & & \\
\hline Baudrand, 2010 [9] & $\mathrm{n}=49$ & & & & & & \\
\hline & Female: 35 (71) & $11(22.4)$ & $49(100)$ & $42 \pm 6.1$ & $31(63.3)$ & $\begin{array}{l}r=0.48, P=0.005 \\
\text { with fasting insulin }\end{array}$ & N/D \\
\hline & Age: $42.2 \pm 10.1$ & & & & & & \\
\hline & Ethnicity: N/D & & & & & & \\
\hline Baudrand, 2011 [11] & $n=41$ & & & & & & \\
\hline & Female: 29 (70) & $6(14.6)$ & $41(100)$ & $36.5(36.5-47.0)$ & $\mathrm{N} / \mathrm{D}$ & $r=0.37, P=0.025$ & N/D \\
\hline & Age: $41.0(34.0-47.5)$ & & & & & . & \\
\hline & Ethnicity: N/D & & & & & & \\
\hline Gyllenhammer, 2014 [12] & $n=36$ & & & & & & \\
\hline & Female: 23 (63.9) & $\mathrm{N} / \mathrm{D}$ & $36(100)$ & $35.2 \pm 0.6$ & $\mathrm{~N} / \mathrm{D}$ & $N / D$ & $r=-0.574, P=0.140$ with \\
\hline & Age: $21.5 \pm 0.4$ & & & & & & \\
\hline & $\begin{array}{l}\text { Ethnicity: } 16 \text { African- } \\
\text { Americans }\end{array}$ & & & & & & \\
\hline & 20 Hispanics & & & & & & \\
\hline
\end{tabular}

Data expressed as absolute and relative frequencies or mean \pm standard deviation or median (p25 - p75) as appropriate. T2DM: type 2 diabetes mellitus; BMI: body mass index; MetS: metabolic syndrome; VAT: visceral adipose tissue; SAT: subcutaneous adipose tissue; N/D: not described; AUC: area under the curve in oral glucose tolerance test; NS: non-significant. 
Table 3 Relationship between HSD11B1 gene expression in abdominal adipose tissue and obesity: studies and subjects' characteristics

\begin{tabular}{|c|c|c|c|c|c|c|c|}
\hline $\begin{array}{l}\text { Author, } \\
\text { year }\end{array}$ & $\begin{array}{l}\text { Subjects' } \\
\text { characteristics }\end{array}$ & T2DM & Obesity & BMI $\left(\mathrm{kg} / \mathrm{m}^{2}\right)$ & MetS & Expression in VAT & Expression in SAT \\
\hline \multirow[t]{4}{*}{ Paulmyer-Lacroix, 2002 [21] } & $\mathrm{n}: 30$ & & & & & & \\
\hline & Female: 27 (90) & $\mathrm{N} / \mathrm{D}$ & $18(60)$ & $33.8 \pm 5.3$ & N/D & $\mathrm{N} / \mathrm{D}$ & $\mathrm{BMI}<25 \mathrm{~kg} / \mathrm{m}^{2}: 1.6(\mathrm{HA})$ \\
\hline & Age: $37.8 \pm 11.2$ & & & & & & $\mathrm{BMI} \geq 30 \mathrm{~kg} / \mathrm{m}^{2}: 4.0(\mathrm{HA})^{\mathrm{a}}$ \\
\hline & Ethnicity: N/D & & & & & & \\
\hline \multirow[t]{4}{*}{ Tomlinson, 2002 [26] } & n: 32 & & & & & & \\
\hline & Female: 32 (100) & $0(0)$ & $11(34.4)$ & $28.1 \pm 0.7$ & N/D & $\mathrm{BMI}<30 \mathrm{~kg} / \mathrm{m}^{2}: 15.5 \pm 0.3$ & $\mathrm{BMl}<30 \mathrm{~kg} / \mathrm{m}^{2}: 15.5 \pm 0.4$ (n-fold) \\
\hline & Age: $43(28-65)$ & & & & & (n-fold) & \\
\hline & Ethnicity: N/D & & & & & $\begin{array}{l}\mathrm{BMI} \geq 30 \mathrm{~kg} / \mathrm{m}^{2}: 14.8 \pm 0.5 \\
(\mathrm{n} \text {-fold })\end{array}$ & $\mathrm{BMI} \geq 30 \mathrm{~kg} / \mathrm{m}^{2}: 15.1 \pm 0.5$ (n-fold) \\
\hline \multirow[t]{4}{*}{ Wake, 2003 [27] } & n: 32 & & & & & & \\
\hline & Female: $16(50.0)$ & $0(0)$ & N/D & $25.9 \pm 0.9$ & N/D & N/D & $r=0.63$ with $\mathrm{BMI}^{\mathrm{c}}$ \\
\hline & Age: $55.5 \pm 3.0$ & & & & & & \\
\hline & Ethnicity: Caucasian & & & & & & \\
\hline \multirow[t]{4}{*}{ Lindsay, 2003 [13] } & n: 31 & & & & & & \\
\hline & Female: 14 (45.2) & N/D & N/D & $35.4 \pm 7.4$ & N/D & N/D & $r=0.34$ with $B M I^{a}$ \\
\hline & Age: $28.8 \pm 7.7$ & & & & & & \\
\hline & Ethnicity: 12 Caucasian & & & & & & \\
\hline \multirow[t]{4}{*}{ Engeli, 2004 [16] } & n: $70 \quad 19$ Pima Indian & & & & & & \\
\hline & Female: 70 (100) & $0(0)$ & $14(20)$ & $29.0 \pm 3.1$ & N/D & N/D & $\mathrm{BMl}<25 \mathrm{~kg} / \mathrm{m}^{2}: 0.5(\mathrm{AU})$ \\
\hline & Age: $57 \pm 4$ & & & & & & $\mathrm{BMI} \geq 25 \mathrm{~kg} / \mathrm{m}^{2}: 0.9(\mathrm{AU})^{\mathrm{a}}$ \\
\hline & Ethnicity: Caucasian & & & & & & \\
\hline \multirow[t]{6}{*}{ Goedecke, 2006 [11] } & n: 26 & & & & & & \\
\hline & Female: 26 (100) & $0(0)$ & N/D & $27.2 \pm 0.9$ & N/D & $r=0.35$ with VAT volume & $r=0.57$ with VAT volume ${ }^{a}$ \\
\hline & Age: $41 \pm 2.0$ & & & & & & \\
\hline & Ethnicity: 11 Caucasian & & & & & & \\
\hline & 8 Mixed race & & & & & & \\
\hline & 7 Black & & & & & & \\
\hline \multirow[t]{3}{*}{ Desbriere, 2006 [15] } & n: 22 & & & & & & \\
\hline & Female: 22 (100) & $0(0)$ & $12(54.5)$ & $30.2 \pm 3.8$ & N/D & $\mathrm{BMI}<25 \mathrm{~kg} / \mathrm{m}^{2}: 0.5(\mathrm{AU})$ & $\mathrm{BMI}<25 \mathrm{~kg} / \mathrm{m}^{2}: 0.5(\mathrm{AU})$ \\
\hline & Age: $36.2 \pm 7.5$ & & & & & $\mathrm{BMI} \geq 30 \mathrm{~kg} / \mathrm{m}^{2}: 2.2(\mathrm{AU})^{\mathrm{a}}$ & $\mathrm{BMI} \geq 30 \mathrm{~kg} / \mathrm{m}^{2}: 3.6(\mathrm{AU})^{\mathrm{a}}$ \\
\hline
\end{tabular}

Ethnicity: N/D 
Table 3 Relationship between HSD11B1 gene expression in abdominal adipose tissue and obesity: studies and subjects' characteristics (Continued)

\begin{tabular}{|c|c|c|c|c|c|c|c|}
\hline \multirow[t]{4}{*}{ Mariniello, 2006 [19] } & $\mathrm{n}: 24$ & & & & & & \\
\hline & Female: 17 (70.8) & \multirow[t]{3}{*}{ N/D } & \multirow[t]{3}{*}{$8(33.3)$} & \multirow[t]{3}{*}{$34 \pm 3.4$} & \multirow[t]{3}{*}{ N/D } & $\mathrm{BMI}<30$ kg/m²: 1 (n-fold) & \multirow[t]{3}{*}{ N/D } \\
\hline & Age: $42 \pm 10$ & & & & & $\mathrm{BMI} \geq 30 \mathrm{~kg} / \mathrm{m}^{2}: 13(\mathrm{n} \text {-fold })^{\mathrm{b}}$ & \\
\hline & Ethnicity: N/D & & & & & & \\
\hline \multirow[t]{4}{*}{ Alberti, 2007 [6] } & $\mathrm{n}: 62$ & & & & & & \\
\hline & Female: 52 (83.9) & \multirow[t]{3}{*}{$12(19.4)$} & \multirow[t]{3}{*}{$62(100.0)$} & \multirow[t]{3}{*}{$37.4 \pm 5.1$} & \multirow[t]{3}{*}{$25(40.3)$} & \multirow{3}{*}{$\begin{array}{l}\mathrm{BMI} \geq 30 \mathrm{~kg} / \mathrm{m}^{2}: 2.1 \pm 0.9 \\
(\mathrm{AU})\end{array}$} & \multirow[t]{3}{*}{$\mathrm{BMI} \geq 30 \mathrm{~kg} / \mathrm{m}^{2}: 1.4 \pm 0.8(\mathrm{AU})$} \\
\hline & Age: $44.4 \pm 11.1$ & & & & & & \\
\hline & Ethnicity: N/D & & & & & & \\
\hline \multirow[t]{4}{*}{ Makkonen, 2007 [18] } & n: 20 & & & & & & \\
\hline & Female: 20 (100) & \multirow[t]{3}{*}{$0(0)$} & \multirow[t]{3}{*}{$10(50)$} & \multirow[t]{3}{*}{$28.7 \pm 1.4$} & \multirow[t]{3}{*}{ N/D } & \multirow[t]{3}{*}{ N/D } & $\mathrm{BMI}<25 \mathrm{~kg} / \mathrm{m}^{2}: 0.3 \pm 0.1$ (n-fold) \\
\hline & Age: $36.5 \pm 3.5$ & & & & & & $\mathrm{BMI} \geq 25 \mathrm{~kg} / \mathrm{m}^{2}: 0.6 \pm 0.1(\mathrm{n} \text {-fold })^{\mathrm{a}}$ \\
\hline & Ethnicity: Caucasian & & & & & & \\
\hline \multirow[t]{4}{*}{ Michailidou, 2007 [20] } & $\mathrm{n}: 21$ & & & & & & \\
\hline & Female: 21 (100) & \multirow[t]{3}{*}{ N/D } & \multirow[t]{3}{*}{ N/D } & \multirow[t]{3}{*}{$32.7 \pm 1.5$} & \multirow[t]{3}{*}{ N/D } & \multirow[t]{3}{*}{$r=0.57$ with $\mathrm{BMI}^{\mathrm{c}}$} & \multirow[t]{3}{*}{$r=0.58$ with $\mathrm{BMI}^{\mathrm{a}}$} \\
\hline & Age: $35 \pm 1$ & & & & & & \\
\hline & Ethnicity: Caucasian & & & & & & \\
\hline \multirow[t]{4}{*}{ Paulsen, 2007 [22] } & n: 40 & & & & & & \\
\hline & Female: 20 (50) & \multirow[t]{3}{*}{$0(0)$} & \multirow[t]{3}{*}{$20(50)$} & $34.7 \pm 3.8$ & $0(0)$ & $\begin{array}{l}\mathrm{BMI}<30 \mathrm{~kg} / \mathrm{m}^{2}: 0.01 \\
\text { (n-fold) }\end{array}$ & $\mathrm{BMI}<30 \mathrm{~kg} / \mathrm{m}^{2}: 0.01$ (n-fold) \\
\hline & Age: $41.3 \pm 9.8$ & & & & & $\mathrm{BMI} \geq 30 \mathrm{~kg} / \mathrm{m}^{2}: 0.04$ & $\mathrm{BMI} \geq 30 \mathrm{~kg} / \mathrm{m}^{2}: 0.05(\mathrm{n} \text {-fold })^{\mathrm{a}}$ \\
\hline & Ethnicity: N/D & & & & & $(n-f o l d)^{a}$ & \\
\hline Baudrand, 2010 [9] & $n=49$ & & & & & & \\
\hline & Female: 35 (71) & $11(22.4)$ & $49(100)$ & $42 \pm 6.1$ & $31(63.3)$ & N/D & N/D \\
\hline & Age: $42.2 \pm 10.1$ & & & & & & \\
\hline & Ethnicity: N/D & & & & & & \\
\hline Muñoz, 2009 [8] & n: 32 & & & & & & \\
\hline & Female: 21 (65.6) & $6(18.8)$ & $32(100.0)$ & $36.7 \pm 3.8$ & $16(50.0)$ & $\mathrm{BMI} \geq 30 \mathrm{~kg} / \mathrm{m}^{2}: 7.8$ & $\mathrm{BMI} \geq 30 \mathrm{~kg} / \mathrm{m}^{2}: 11.4(6.2-19.8)(\mathrm{AU})$ \\
\hline & Age: $40.2 \pm 12.3$ & & & & & $\begin{array}{l}(4 . /-11.8) \\
(\mathrm{AU})\end{array}$ & \\
\hline & Ethnicity: N/D & & & & & & \\
\hline Simonyte, 2009 [24] & $\mathrm{n}: 27$ & $3(9.7)$ & $27(100)$ & $44.6 \pm 4.5$ & N/D & $\mathrm{BMI} \geq 30 \mathrm{~kg} / \mathrm{m}^{2}: 11.2 \pm 4.9$ & $\mathrm{BMI} \geq 30 \mathrm{~kg} / \mathrm{m}^{2}: 14.1 \pm 6.4(\mathrm{AU})$ \\
\hline & Female: 27 (100) & & & & & & \\
\hline & Age: $41 \pm 8.5$ & & & & & & \\
\hline & Ethnicity: Caucasian & & & & & & \\
\hline
\end{tabular}


Table 3 Relationship between HSD11B1 gene expression in abdominal adipose tissue and obesity: studies and subjects' characteristics (Continued)

\begin{tabular}{|c|c|c|c|c|c|c|c|}
\hline \multirow[t]{4}{*}{ Zha, 2009 [28] } & n: 35 & & & & & & \\
\hline & Female: 17 (49) & $0(0)$ & $15(50)$ & $25.5 \pm 1.5$ & N/D & $\mathrm{BMI}<25 \mathrm{~kg} / \mathrm{m}^{2}: 1.0(\mathrm{AU})$ & $\mathrm{BMI}<25 \mathrm{~kg} / \mathrm{m}^{2}: 1.2(\mathrm{AU})$ \\
\hline & Age: $49.5 \pm 12.5$ & & & & & $\mathrm{BMI} \geq 25 \mathrm{~kg} / \mathrm{m}^{2}: 1.5(\mathrm{AU})^{\mathrm{c}}$ & $\mathrm{BMI} \geq 25 \mathrm{~kg} / \mathrm{m}^{2}: 1.5(\mathrm{AU})^{\mathrm{c}}$ \\
\hline & Ethnicity: Chinese & & & & & & \\
\hline \multirow{4}{*}{ Svendsen, 2009 [25] } & n: 24 & & & & & & \\
\hline & Female: 24 (100) & N/D & $16(66.7)$ & $30.0 \pm 2.7$ & N/D & $N / D$ & $\mathrm{BMl}<25 \mathrm{~kg} / \mathrm{m}^{2}: 0.09(0.03-0.60)$ \\
\hline & Age: $30.7 \pm 4.7$ & & & & & & $(\mathrm{AU})$ \\
\hline & Ethnicity: N/D & & & & & & $\begin{array}{l}\mathrm{BMI} \geq 25 \mathrm{~kg} / \mathrm{m}^{2}: 0.25(0.09-0.84) \\
(\mathrm{AU})^{\mathrm{a}}\end{array}$ \\
\hline \multirow[t]{4}{*}{ Simonyte, 2010 [23] } & $\mathrm{n}: 17$ & & & & & & \\
\hline & Female: 17 (100) & N/D & $17(100)$ & $44.4 \pm 4.4$ & N/D & $N / D$ & $r=0.53$ with $W C^{a}$ \\
\hline & Age: N/D & & & & & & \\
\hline & Ethnicity: N/D & & & & & & \\
\hline \multirow[t]{4}{*}{ Baudrand, 2011 [10] } & $n=41$ & & & & & & \\
\hline & Female: 29 (70) & $6(14.6)$ & $41(100)$ & $36.5(36.5-47.0)$ & N/D & $\mathrm{N} / \mathrm{D}$ & N/D \\
\hline & $\begin{array}{l}\text { Age: } 41.0 \\
(34.0-47.5)\end{array}$ & & & & & & \\
\hline & Ethnicity: N/D & & & & & & \\
\hline \multirow[t]{4}{*}{ Michalaki, 2012 [7] } & $n=27$ & & & & & & $\mathrm{BMI}<25 \mathrm{~kg} / \mathrm{m}^{2}: \mathrm{N} / \mathrm{D}$ \\
\hline & Female: 27 (100) & $2(7.4)$ & $19(70.4)$ & $46.1 \pm 6.6$ & $11(40.7)$ & $\begin{array}{l}\mathrm{BMl}<25 \mathrm{~kg} / \mathrm{m}^{2}: 27.8 \pm 16 \\
(\mathrm{AU})\end{array}$ & \\
\hline & Age: $37.3 \pm 9.7$ & & & & & (AU) & \\
\hline & Ethnicity: N/D & & & & & $\begin{array}{l}B M I \geq 30 \mathrm{~kg} / \mathrm{m}^{2}: 81.6 . \pm 46.8 \\
(A \cup)^{\mathrm{a}}\end{array}$ & $\mathrm{BMI} \geq 30 \mathrm{~kg} / \mathrm{m}^{2}: 115.7 . \pm 69.8(\mathrm{AU})$ \\
\hline \multirow[t]{3}{*}{ Leyvraz, 2012 [17] } & $n=35$ & & & & & & \\
\hline & Female: 35 (100) & N/D & $30(85.7)$ & $41.3 \pm 4.6$ & N/D & $\mathrm{BMI}<30 \mathrm{~kg} / \mathrm{m}^{2}: 1.0(0.6-1.4)(\mathrm{AU})$ & $\mathrm{BMl}<30 \mathrm{~kg} / \mathrm{m}^{2}: 1.0(0.8-1.2)(\mathrm{AU})$ \\
\hline & Age: $39.0 \pm 9.0$ & & & & & $\begin{array}{l}\mathrm{BMI} \geq 30 \mathrm{~kg} / \mathrm{m}^{2}: 2.9 \cdot(2.6-3.2) \\
(\mathrm{AU})^{\mathrm{c}}\end{array}$ & $\mathrm{BMI} \geq 30 \mathrm{~kg} / \mathrm{m}^{2}: 2.2(2.1-2.3)(\mathrm{AU})^{\mathrm{c}}$ \\
\hline
\end{tabular}

Data expressed as absolute and relative frequencies, median (interquartile range), or mean \pm standard deviation as appropriate. T2DM: type 2 diabetes mellitus; BMI: body mass index; MetS: metabolic syndrome; VAT: visceral adipose tissue; SAT: subcutaneous adipose tissue; HA: hybridized area/field; AU: arbitrary units; WC: waist circumference. ${ }^{\mathrm{a} P}<0.05$. ${ }^{\mathrm{b}} \mathrm{P}<0.001$. ${ }^{\mathrm{C}} \mathrm{P}<0.01$. $\mathrm{r}=\mathrm{Pears}$ (n)'s correlation coefficient. N/D: not described; NS: non-significant. 
Table 4 Relationship of HSD11B1 gene polymorphic variants with obesity, the metabolic syndrome, and diabetes: studies and subjects' characteristics

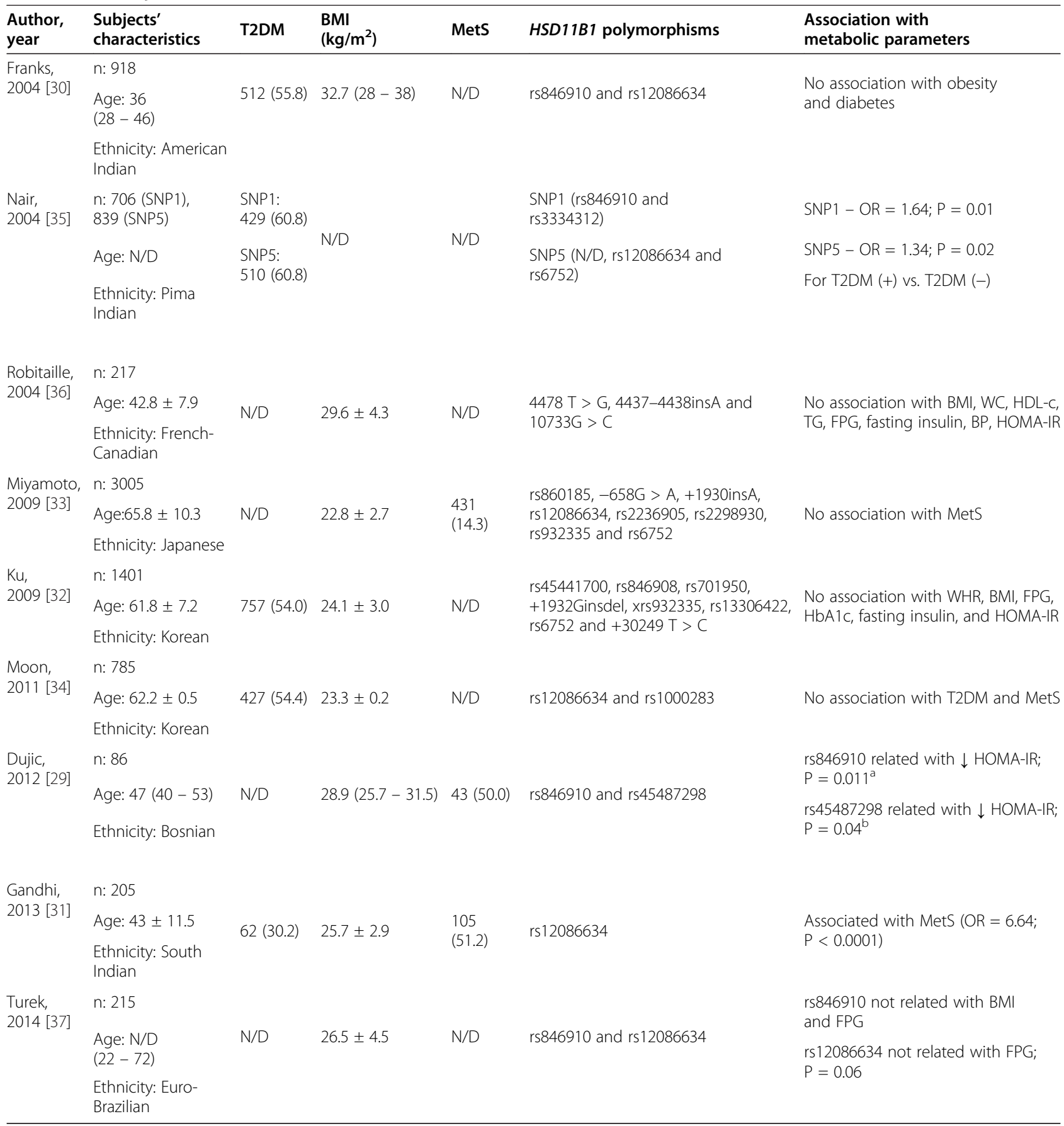

Data expressed as absolute and relative frequencies, median (interquartile range), or mean \pm standard deviation as appropriate. N/D: not described; T2DM: type 2 diabetes mellitus; BMI: body mass index; MetS: metabolic syndrome; WC: waist circumference; HDL-c: HDL cholesterol; TG: triglycerides; FPG: fasting plasma glucose; BP: blood pressure; HOMA-IR: homeostatic model assessment of insulin resistance; HbA1c: glycated hemoglobin A1c; NPG: non-polymorphic group; PG: polymorphic group. in MetS (+) group. ${ }^{\text {in }}$ control group. 
Table 5 Quality scores of studies included in the systematic review

\begin{tabular}{|c|c|c|c|c|c|c|c|}
\hline Author, year & $\begin{array}{l}\text { Case } \\
\text { definition }\end{array}$ & $\begin{array}{l}\text { Representativeness } \\
\text { of the cases }\end{array}$ & $\begin{array}{l}\text { Selection } \\
\text { of controls }\end{array}$ & $\begin{array}{l}\text { Definition } \\
\text { of controls }\end{array}$ & Comparability & $\begin{array}{l}\text { Ascertainment } \\
\text { of exposure }\end{array}$ & Total \\
\hline Alberti, 2007 [6] & + & & + & + & + & ++ & ++++++ \\
\hline Baudrand, 2010 [9] & + & + & & + & & ++ & +++++ \\
\hline Baudrand, 2011 [10] & + & + & & & & + & +++ \\
\hline Desbriere, 2006 [15] & + & & & + & & ++ & ++++ \\
\hline Dujic, 2012 & + & + & + & + & & +++ & +++++++ \\
\hline Engeli, 2004 [16] & + & + & + & + & & +++ & +++++++ \\
\hline Franks, 2004 [30] & + & + & + & & + & +++ & +++++++ \\
\hline Gandhi, 2013 [31] & + & + & + & + & & ++ & ++++++ \\
\hline Goedecke, 2006 [11] & + & + & & & + & + & ++++ \\
\hline Gyllenhammer, 2014 [12] & + & + & & & ++ & + & +++++ \\
\hline $\mathrm{Ku}, 2009$ [32] & + & + & + & + & + & +++ & ++++++++ \\
\hline Leyvraz, 2012 [17] & + & + & & & & + & +++ \\
\hline Lindsay, 2003 [13] & + & + & & & + & ++ & +++++ \\
\hline Makkonen, 2007 [18] & + & & + & + & + & ++ & ++++++ \\
\hline Mariniello, 2006 [19] & + & & + & + & & ++ & +++++ \\
\hline Michailidou, 2007 [20] & + & & & & + & ++ & ++++ \\
\hline Michalaki, 2012 [7] & + & & & + & & ++ & ++++ \\
\hline Miyamoto, 2009 [2009] & + & + & + & & + & ++ & ++++++ \\
\hline Moon, 2011 [34] & + & + & + & + & + & +++ & ++++++++ \\
\hline Munoz, 2009 [8] & + & + & & & & ++ & ++++ \\
\hline Nair, 2004 [35] & + & + & + & & + & +++ & +++++++ \\
\hline Paulmyer-Lacroix, 2002 [21] & + & + & + & + & & +++ & +++++++ \\
\hline Paulsen, 2007 [22] & + & & & + & & ++ & ++++ \\
\hline Robitaille, 2004 [36] & + & & & & & +++ & ++++ \\
\hline Simonyte, 2009 [24] & + & & & & + & ++ & ++++ \\
\hline Simonyte, 2010 [23] & + & + & & & + & + & ++++ \\
\hline Svendsen, 2009 [25] & + & + & + & + & ++ & ++ & ++++++++ \\
\hline Tomlinson, 2002 [26] & + & & & + & & ++ & ++++ \\
\hline Tomlinson, 2008 [14] & + & + & & & ++ & + & +++++ \\
\hline Turek, 2014 [37] & + & + & & & + & ++ & +++++ \\
\hline Wake, 2003 [27] & + & + & & & ++ & + & +++++ \\
\hline Zha, 2009 [28] & + & + & & + & + & ++ & ++++++ \\
\hline
\end{tabular}

Relationship of HSD11B1 genetic polymorphisms with obesity, the metabolic syndrome, and type 2 diabetes mellitus

Nine studies analyzed the association between 26 different $H S D 11 B 1$ polymorphic variants and obesity, MetS, and T2DM (Table 4) [29-37].

Asian populations were assessed in two studies from South Korea [32,34], one study from Japan [33], and one from India [31]. The first study analyzed 757 individuals with and 644 without T2DM [32], and did not find associations between any of four HSD11B1 polymorphisms with T2DM or MetS. In another study, 427 individuals with and 358 without T2DM were analyzed and no relationship was found between two HSD11B1 polymorphic variants and T2DM or MetS [34]. In an urban cohort of 3,005 Japanese individuals, seven HSD11B1 polymorphic variants were assessed and no association with MetS was found [33]. Another study, conducted in 217 French-Canadian men, also failed to find any association between $H S D 11 B 1$ polymorphisms and MetS [36]. Finally, in a study of 918 Native Americans of the Gila River Indian Community of Arizona, polymorphic variants of this gene were not associated with T2DM or obesity [30]. 
In contrast with these negative results, a study conducted in India found a significant risk of MetS in subjects with the HSD11B1 polymorphism rs12086634 [31]. Another study found a significant risk of T2DM, but not of obesity, in Pima Indians with the HSD11B1 polymorphic variants rs846910 and rs12086634 [35]. Conversely, another study found that polymorphism rs846910 was associated with decreased insulin resistance (decreased HOMA-IR) in subjects with MetS, and that the rs45487298 polymorphism was associated with similar findings in control subjects without MetS [29]. Finally, it was shown no relationship between rs846910 with BMI or fasting plasma glucose levels in 215 Euro-Brazilian descendants. The authors of the same study concluded that rs12086634 was related with fasting plasma glucose levels in women with a borderline p-value $(P=0.06)$. However, the sample had limited statistical power, there was no statistical correction for multiple comparisons and no clear definition of what was considered a statistical difference in the methodology section [37].

\section{Discussion}

This systematic review included 32 studies which analyzed the potential relationship of abdominal adipose HSD11B1 gene expression in human and its polymorphic variants with MetS, T2DM, and obesity.

The relationship between HSD11B1 expression in abdominal adipose tissue and MetS was analyzed in three studies with distinct population profiles. They did not point out to a relationship between abdominal SAT and VAT HSD11B1 expression and MetS. However, they were not well designed to answer this question since this was not their main objectives. As a result, these studies might not have enough power to detect differences in HSD11B1 expression in abdominal SAT and VAT comparing groups of obese subjects with and without MetS without having a lean control group for comparison, as what happened in two of these studies. In the third study that we were able to review, lean control subjects had a significantly lower abdominal VAT HSD11B1 expression than those presenting the MetS, strengthening this direction of thought. It is our opinion that this does not mean that there is no relationship between abdominal adipose tissue HSD11B1 and MetS. In other studies it has been shown that a possible relationship between HSD11B1 enzymatic activity and the MetS exists. It also suggests that this enzymatic activity is more linked to MetS than its enzymatic expression [13,38,39]. In another study, increased omental HSD11B1 activity in women submitted to a gynecological surgery was associated with larger omental adipocytes, increased lipolysis and lipoprotein lipase activity, decreased high-density lipoprotein cholesterol and adiponectin levels, and an increased homeostasis model assessment of insulin resistance index compared to those with decreased omental activity of this enzyme [38]. Additionally, HSD11B1 activity was more related with fasting glucose, insulin, BMI and waist circumference than HDS11B1 expression in abdominal SAT in Pima Indians and Caucasians [13]. Moreover, in a study where $H S D 11 B 1$ activity was indirectly studied by a ratio of urinary cortisone to cortisol metabolites, it has been shown a failure to down-regulate HSD11B1 activity in subjects with diabetes compared to normal controls which might contribute to the underlying pathogenesis of the MetS. The lower prevalence of T2DM in the studies analyzed by us may have biased our findings and may explain, in part, why we were not able to find this link [39]. Therefore, no relationship between HSD11B1 gene expression in abdominal adipocytes and MetS can be conclusively established on the basis of the current literature.

A relationship between HSD11B1 expression in abdominal adipose tissue and IR, pancreatic $\beta$-cell function, fasting glucose, and T2DM were analyzed in eight studies of populations with different ethnic backgrounds [6,8-14]. Their results have shown that increasing HSD11B1 expression in abdominal VAT and SAT is associated with IR in 2 studies, impaired $\beta$-cell dysfunction in one study and increased fasting plasma glucose levels in 4 studies (Table 2). Additionally, two studies have shown that HSD11B1 expression in abdominal SAT is greater in those with impaired glucose tolerance and T2DM compared with normal controls. Although it is not clear to define the specific compartment of abdominal adipose tissue in which HSD11B1 expression is mostly associated with metabolic abnormalities of glucose metabolism, these studies suggest that the expression of this gene in abdominal SAT and VAT is related with abnormalities of glucose metabolism and may play a role in the development of T2DM.

The relationship between $H S D 11 B 1$ expression in abdominal adipose tissue and markers of central and general obesity was analyzed in 21 studies. While six studies did not find any association between HSD11B1 expression in abdominal adipose tissue and obesity, fifteen studies found that a greater expression of this gene in VAT and/or SAT was associated with increased BMI, waist circumference, and body fat percentage. The summary data have shown that HSD11B1 expression in abdominal SAT and VAT is related with central obesity and body size. Unfortunately, we were not able to perform a meta-analysis with the summary of these data, since the studies varies in their way to express BMI (absolute number, lean vs overweight vs obesity, lean controls vs excessive weight), abdominal compartment HSD11B1 expression (SAT, VAT or SAT + VAT) and different methodologies to quantify this expression (hybridized area/field vs relative curve vs $\Delta \Delta_{\mathrm{ct}}$ ).

Our collected data suggest that $H S D 11 B 1$ polymorphic variants are not associated with MetS, obesity, or T2DM in most studies [30,33-37]. However, in one study, two HSD11B1 polymorphisms (rs846910 and rs12086634) in 
linkage with three other polymorphisms were found to be associated with decreased insulin sensitivity, increased plasma glucose levels, and T2DM in Pima Indians [35]. Additionally, the rs12086634 polymorphism was found to be associated with MetS in a study conducted in India [31]. The polymorphic variant rs 846910 is located in the promoter region near exon 1 of the HSD11B1 gene. Carriers of this SNP exhibit increased enzyme activity, which is in agreement with these findings. However, rs12086634 is located in intron 3, and carriers of this polymorphic variant exhibit decreased enzyme activity, which cannot be explained by our findings regarding this SNP. Additionally, the polymorphic variant rs846910 and another one, rs45487298 (SNP localized in intron 3 of the HSD11B1 gene and related to decreased enzyme expression), were associated respectively with increased insulin sensitivity in subjects with and without MetS in another study [29]. Due to the small sample size to take in account their results $(n=86)$, it is necessary more solid data to take in account these results. Additionally, since polymorphic variants of $H S D 11 B 1$ that change its expression and activity are expected to have a major role in modulating insulin sensitivity and adipose tissue proliferation through regulation of intra-adipocyte cortisol production, the presence of these polymorphic variants would be expected to be associated with obesity as well, which was not demonstrated in the present review [35]. None of studies included in this review assessed how these polymorphic variants affect $H S D 11 B 1$ functionality. Since HSD11B1 activity is dependent on the provision of NADPH by the co-localized enzyme hexose6-phosphate dehydrogenase (H6PD), polymorphic variants

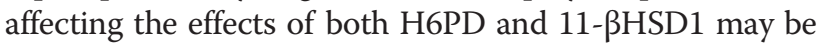
necessary to exert an effect on enzymatic activity, resulting in metabolic abnormalities or a healthy metabolic profile.

\section{Conclusions}

In conclusion, our systematic review have shown that expression of the HSD11B1 gene in intra-abdominal adipose tissue is probably related to abnormalities of glucose metabolism, T2DM and obesity, with consistent findings across different studies. We were not able to find that expression of the HSD11B1 gene in intra-abdominal adipose tissue is related with MetS. Finally, the relationship between polymorphic variants of HSD11B1 with MetS in subjects from India and T2DM in Pima Indians, but not in studies performed in several other populations, suggests that $H S D 11 B 1$ polymorphisms may have a small role in the development of these metabolic abnormalities only in these susceptible populations.

\footnotetext{
Abbreviations

11-ßHSD1: Hydroxysteroid (11-beta) dehydrogenase type 1; IR: Insulin resistance; MetS: Metabolic syndrome; T2DM: Type 2 diabetes mellitus; BMI: Body mass index; NOS: New Castle-Ottawa Scale; SAT: Abdominal
}

subcutaneous adipose tissue; VAT: Abdominal visceral adipose tissue; H6PD: Hexose-6-phosphate dehydrogenase.

\section{Competing interests}

The authors declare that they have no competing interests.

\section{Authors' contributions}

FVdN: conception and design of study, acquisition, analysis and interpretation of data, draft of manuscript. VP: acquisition, analysis and interpretation of data. MAB: acquisition, analysis and interpretation of data. ADVF: interpretation of data, revision of draft. DC: conception and design of study, revision of draft. FG: conception and design of study, interpretation of data, revision of draft and final approval of the version to be published. All authors read and approved the final manuscript.

\section{Acknowledgements}

This study was supported by grants from Fundação de Amparo à Pesquisa do Estado do Rio Grande do Sul (FAPERGS), Conselho Nacional de

Desenvolvimento Científico e Tecnológico (CNPq), and Fundo de Incentivo à Pesquisa e Eventos do Hospital de Clínicas de Porto Alegre (FIPE-HCPA).

Received: 11 January 2015 Accepted: 21 April 2015

Published online: 28 April 2015

\section{References}

1. Tomlinson JW, Moore JS, Clark PM, Holder G, Shakespeare L, Stewart PM. Weight loss increases 11 beta-hydroxysteroid dehydrogenase type 1 expression in human adipose tissue. J Clin Endocrinol Metab. 2004;89(6):2711-6.

2. Masuzaki H, Paterson J, Shinyama H, Morton NM, Mullins JJ, Seckl JR, et al. A transgenic model of visceral obesity and the metabolic syndrome. Science. 2001;294(5549):2166-70.

3. Kotelevtsev Y, Holmes MC, Burchell A, Houston PM, Schmoll D, Jamieson P, et al. 11 beta-hydroxysteroid dehydrogenase type 1 knockout mice show attenuated glucocorticoid-inducible responses and resist hyperglycemia on obesity or stress. Proc Natl Acad Sci U S A. 1997;94(26):14924-9.

4. Stimson RH, Walker BR. Glucocorticoids and 11 beta-hydroxysteroid dehydrogenase type 1 in obesity and the metabolic syndrome. Minerva Endocrinol. 2007;32(3):141-59.

5. Stang A. Critical evaluation of the Newcastle-Ottawa scale for the assessment of the quality of nonrandomized studies in meta-analyses. Eur J Epidemiol. 2010;25(9):603-5

6. Alberti L, Girola A, Gilardini L, Conti A, Cattaldo S, Micheletto G, et al. Type 2 diabetes and metabolic syndrome are associated with increased expression of 11 beta-hydroxysteroid dehydrogenase 1 in obese subjects. Int J Obes. 2007;31(12):1826-31.

7. Michalaki M, Kyriazopoulou V, Antonacopoulou A, Koika V, Nikolaou M, Tsoukas A, et al. The expression of omental 11 beta-HSD1 is not increased in severely obese women with metabolic syndrome. Obes Facts. 2012;5(1):104-11.

8. Munoz R, Carvajal C, Escalona A, Boza C, Perez G, Ibanez L, et al. 11 betahydroxysteroid dehydrogenase type 1 is overexpressed in subcutaneous adipose tissue of morbidly obese patients. Obes Surg. 2009;19(6):764-70.

9. Baudrand R, Carvajal CA, Riquelme A, Morales M, Solis N, Pizarro M, et al. Overexpression of 11 beta-hydroxysteroid dehydrogenase type 1 in hepatic and visceral adipose tissue is associated with metabolic disorders in morbidly obese patients. Obes Surg. 2010;20(1):77-83.

10. Baudrand R, Dominguez JM, Carvajal CA, Riquelme A, Campino C, Macchiavello S, et al. Overexpression of hepatic 5alpha-reductase and 11 beta-hydroxysteroid dehydrogenase type 1 in visceral adipose tissue is associated with hyperinsulinemia in morbidly obese patients. Metabolism. 2011;60(12):1775-80.

11. Goedecke JH, Wake DJ, Levitt NS, Lambert EV, Collins MR, Morton NM, et al. Glucocorticoid metabolism within superficial subcutaneous rather than visceral adipose tissue is associated with features of the metabolic syndrome in South African women. Clin Endocrinol (Oxf). 2006;65(1):81-7.

12. Gyllenhammer LE, Alderete TL, Mahurka S, Allayee H, Goran MI. Adipose tissue 11 betaHSD1 gene expression, betacell function and ectopic fat in obese African Americans versus Hispanics. Obesity. 2014;22(1):14-8.

13. Lindsay RS, Wake DJ, Nair S, Bunt J, Livingstone DE, Permana PA, et al. Subcutaneous adipose 11 beta-hydroxysteroid dehydrogenase type 1 activity and messenger ribonucleic acid levels are associated with adiposity and 
insulinemia in Pima Indians and Caucasians. J Clin Endocrinol Metab. 2003;88(6):2738-44.

14. Tomlinson JW, Finney J, Gay C, Hughes BA, Hughes SV, Stewart PM. Impaired glucose tolerance and insulin resistance are associated with increased adipose 11 beta-hydroxysteroid dehydrogenase type 1 expression and elevated hepatic 5alpha-reductase activity. Diabetes. 2008;57(10):2652-60.

15. Desbriere R, Vuaroqueaux V, Achard V, Boullu-Ciocca S, Labuhn M, Dutour A, et al. 11 beta-hydroxysteroid dehydrogenase type 1 mRNA is increased in both visceral and subcutaneous adipose tissue of obese patients. Obesity. 2006;14(5):794-8.

16. Engeli S, Bohnke J, Feldpausch M, Gorzelniak K, Heintze U, Janke J, et al. Regulation of 11 beta-HSD genes in human adipose tissue: influence of central obesity and weight loss. Obes Res. 2004;12(1):9-17.

17. Leyvraz C, Verdumo C, Suter M, Paroz A, Calmes JM, Marques-Vidal PM, et al. Changes in gene expression profile in human subcutaneous adipose tissue during significant weight loss. Obes Facts. 2012;5(3):440-51.

18. Makkonen J, Westerbacka J, Kolak M, Sutinen J, Corner A, Hamsten A, et al. Increased expression of the macrophage markers and of 11 beta-HSD-1 in subcutaneous adipose tissue, but not in cultured monocyte-derived macrophages, is associated with liver fat in human obesity. Int J Obes. 2007:31(10):1617-25.

19. Mariniello B, Ronconi V, Rilli S, Bernante P, Boscaro M, Mantero F, et al. Adipose tissue 11 beta-hydroxysteroid dehydrogenase type 1 expression in obesity and cushings syndrome. Eur J Endocrinol. 2006;155(3):435-41.

20. Michailidou Z, Jensen MD, Dumesic DA, Chapman KE, Seckl JR, Walker BR, et al. Omental 11 beta-hydroxysteroid dehydrogenase 1 correlates with fat cell size independently of obesity. Obesity. 2007;15(5):1155-63.

21. Paulmyer-Lacroix O, Boullu S, Oliver C, Alessi MC, Grino M. Expression of the mRNA coding for 11 beta-hydroxysteroid dehydrogenase type 1 in adipose tissue from obese patients: an in situ hybridization study. J Clin Endocrinol Metab. 2002;87(6):2701-5.

22. Paulsen SK, Pedersen SB, Fisker S, Richelsen B. 11Beta-HSD type 1 expression in human adipose tissue: impact of gender, obesity, and fat localization. Obesity. 2007;15(8):1954-60.

23. Simonyte K, Olsson T, Naslund I, Angelhed JE, Lonn L, Mattsson C, et al. Weight loss after gastric bypass surgery in women is followed by a metabolically favorable decrease in 11 beta-hydroxysteroid dehydrogenase 1 expression in subcutaneous adipose tissue. J Clin Endocrinol Metab. 2010;95(7):3527-31.

24. Simonyte K, Rask E, Naslund I, Angelhed JE, Lonn L, Olsson T, et al. Obesity is accompanied by disturbances in peripheral glucocorticoid metabolism and changes in FA recycling. Obesity. 2009;17(11):1982-7.

25. Svendsen PF, Madsbad S, Nilas L, Paulsen SK, Pedersen SB. Expression of 11 beta-hydroxysteroid dehydrogenase 1 and 2 in subcutaneous adipose tissue of lean and obese women with and without polycystic ovary syndrome. Int J Obes. 2009;33(11):1249-56.

26. Tomlinson JW, Sinha B, Bujalska I, Hewison M, Stewart PM. Expression of 11 beta-hydroxysteroid dehydrogenase type 1 in adipose tissue is not increased in human obesity. J Clin Endocrinol Metab. 2002;87(12):5630-5.

27. Wake DJ, Rask E, Livingstone DE, Soderberg S, Olsson T, Walker BR. Local and systemic impact of transcriptional up-regulation of 11 betahydroxysteroid dehydrogenase type 1 in adipose tissue in human obesity. J Clin Endocrinol Metab. 2003;88(8):3983-8.

28. Zha JM, Di WJ, Zhu T, Xie Y, Yu J, Liu J, et al. Comparison of gene transcription between subcutaneous and visceral adipose tissue in Chinese adults. Endocr J. 2009:56(8):935-44.

29. Dujic T, Bego T, Mlinar B, Semiz S, Malenica M, Prnjavorac B, et al. Association between 11 beta-hydroxysteroid dehydrogenase type 1 gene polymorphisms and metabolic syndrome in Bosnian population. Biochem Med. 2012;22(1):76-85

30. Franks PW, Knowler WC, Nair S, Koska J, Lee YH, Lindsay RS, et al. Interaction between an 11 betaHSD1 gene variant and birth era modifies the risk of hypertension in Pima Indians. Hypertension. 2004;44(5):681-8.

31. Gandhi K, Adhikari P, Basu A, Achappa B. Association between a 11 betahydroxysteroid dehydrogenase type 1 gene polymorphism and metabolic syndrome in a South Indian population. Metab Syndr Relat Disord. 2013;11(6):397-402.

32. Ku YH, Koo BK, Kwak SH, Cho YM, Shin HD, Lee HK, et al. Regulatory effect of common promoter polymorphisms on the expression of the 11 betahydroxysteroid dehydrogenase type 1 gene. Horm Res. 2009;72(1):25-32.

33. Miyamoto Y, Morisaki H, Yamanaka I, Kokubo Y, Masuzaki H, Okayama A, et al. Association study of 11 beta-hydroxysteroid dehydrogenase type 1 gene polymorphisms and metabolic syndrome in urban Japanese cohort. Diabetes Res Clin Pract. 2009;85(2):132-8.

34. Moon SS, Lee YS, Kim JG, Kim SW, Jeong JY, Jeon EJ, et al. Relationship of 11 beta-hydroxysteroid dehydrogenase type 1 and hexose-6-phosphate dehydrogenase gene polymorphisms with metabolic syndrome and type 2 diabetes. Endocr J. 2011:58(11):949-59.

35. Nair S, Lee YH, Lindsay RS, Walker BR, Tataranni PA, Bogardus C, et al. 11 beta-Hydroxysteroid dehydrogenase type 1: genetic polymorphisms are associated with Type 2 diabetes in Pima Indians independently of obesity and expression in adipocyte and muscle. Diabetologia. 2004;47(6):1088-95.

36. Robitaille J, Brouillette C, Houde A, Despres JP, Tchernof A, Vohl MC Molecular screening of the 11 beta-HSD1 gene in men characterized by the metabolic syndrome. Obes Res. 2004;12(10):1570-5.

37. Turek LV, Leite N, Rodrigues Souza RL, Lima JK, Milano GE, Timossi Lda S, et al. Gender-dependent association of HSD11B1 single nucleotide polymorphisms with glucose and HDL-C levels. Genet Mol Biol. 2014;37(3):490-5.

38. Veilleux A, Rheaume C, Daris M, Luu-The V, Tchernof A. Omental adipose tissue type 111 beta-hydroxysteroid dehydrogenase oxoreductase activity, body fat distribution, and metabolic alterations in women. J Clin Endocrinol Metab. 2009;94(9):3550-7.

39. Valsamakis G, Anwar A, Tomlinson JW, Shackleton CH, McTernan PG, Chetty $\mathrm{R}$, et al. 11 beta-hydroxysteroid dehydrogenase type 1 activity in lean and obese males with type 2 diabetes mellitus. J Clin Endocrinol Metab. 2004;89(9):4755-61.

\section{Submit your next manuscript to BioMed Central and take full advantage of:}

- Convenient online submission

- Thorough peer review

- No space constraints or color figure charges

- Immediate publication on acceptance

- Inclusion in PubMed, CAS, Scopus and Google Scholar

- Research which is freely available for redistribution 